\title{
The ATLAS Muon Trigger vertical slice at LHC startup
}

\section{Sergio Grancagnolo* on behalf of the ATLAS Collaboration}

INFN Lecce and Università del Salento

E-mail: sergio.grancagnolodcern.ch

The ATLAS trigger system has a three-levels structure, implemented to retain interesting physics events, here described for the muon case ("Muon Vertical Slice"). The first level, implemented in a custom hardware, uses measurements from the trigger chambers of the Muon Spectrometer to select muons with high transverse momentum and defines a Region of Interest (RoI) in the detector. RoIs are then processed by a second trigger level, in which fast algorithms run on an online software architecture. Full granularity information from precision chambers is accessed inside RoIs. A third trigger level (Event Filter), using offline-like algorithms and accessing the full event, provide the best possible muon reconstruction/identification and finally confirm or discard the trigger hypothesis formed at earlier levels. Implementation and performance of the full muon trigger slice, together with first events triggered with LHC beams on, are presented.

XII Advanced Computing and Analysis Techniques in Physics Research November 3-7, 2008

Erice, Italy

\footnotetext{
* Speaker.
} 


\section{Introduction}

The ATLAS trigger system is designed to keep high efficiency for interesting events, while rejecting standard model physics low pT events, with a suppression factor of the order of $10^{7}$, producing a final output rate for offline analysis of $200 \mathrm{~Hz}$.

High pT muons are important for many known processes, that can be used for monitoring and calibration $(Z \rightarrow \mu \mu)$ and for several new phenomena predicted at the LHC energy (Higgs, SUSY), therefore the muon trigger system is of primary importance.

The Muon Spectrometer (MS) is the detector dedicated to the identification of muons. It consists of Resistive Plate Chambers (RPC) and Thin Gap Chambers (TGC) for triggering and Monitored Drift Tubes (MDT) and Cathode Strip Chambers (CSC) for precision measurements.

The Muon Vertical Slice consists of three main trigger steps, one hardware, level 1 (LVL1) and two software, level 2 (LVL2) and event filter (EF). Last two compose the High Level Trigger (HLT).

During the run with the first beam, only the LVL1 was inserted in the data taking. The HLT was running in a transparent mode, flagging the events without any rejection. This allows to study the trigger capability without affecting the data taking, and comparing results with respect to the full reconstruction offline.

\section{Level 1 trigger}

LVL1 selects active detector regions (Region of Interest, RoI), in each event, using RPC for $|\eta|<1$ and TGC for $1<|\eta|<2.4$. Coincidence windows are defined on the allowed geometrical roads with their center corresponding to the infinite momentum track.

Six programmable thresholds of the muon $\mathrm{p}_{T}$ are applied: a low $\mathrm{p}_{T}$ scheme (for $6,8,10 \mathrm{GeV} / \mathrm{c}$ ) and a high $\mathrm{p}_{T}(11,20,40 \mathrm{GeV} / \mathrm{c})$ level triggers.

Overall barrel LVL1 acceptance is $83 \%$ for low $\mathrm{p}_{T}$, and $79 \%$ for high $\mathrm{p}_{T}$ particles, while it is close to 1 for the endcap. The rates depend on the machine luminosity: at the low luminosity of $10^{33} \mathrm{~cm}^{-2} \mathrm{~s}^{-1}$ with the low $\mathrm{p}_{T}$ threshold, the expected rate is about $11 \mathrm{kHz}$, both for the barrel and the endcap, while at the high luminosity of $10^{34} \mathrm{~cm}^{-2} \mathrm{~s}^{-1}$ with the high $\mathrm{p}_{T}$ threshold, the expected rate is about $2 \mathrm{kHz}$ for the barrel and close to $8 \mathrm{kHz}$ for the endcap.

A special setup was configured for taking data during first beam, similar to the one used during cosmic runs. The trigger window was fully open, allowing for tracks not pointing to the interaction region. An example of an event triggered during the first beam run is presented in figure 1

\section{Level 2 trigger}

Many different software algorithms compose the LVL2 trigger. Core algorithm is muFast, that confirms/rejects LVL1 result and refine muon pT evaluation, using MDT precision measurements.

The following steps are executed within a 40 ms processing time: "global pattern recognition", involving trigger chambers and positions of MDT tubes (without using drift time); "local segment reconstruction" involving drift time measurements for each station; fast " $\mathrm{p}_{T}$ estimate" via a lookup-table. 


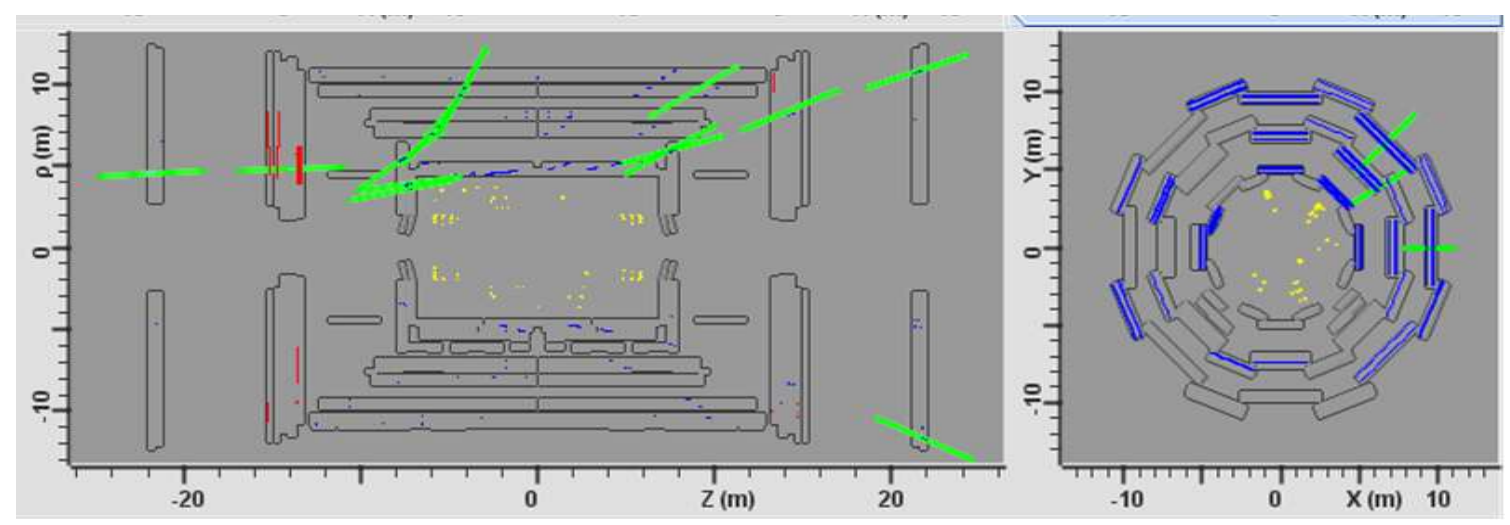

Figure 1: LVL1 triggered event during first beam.
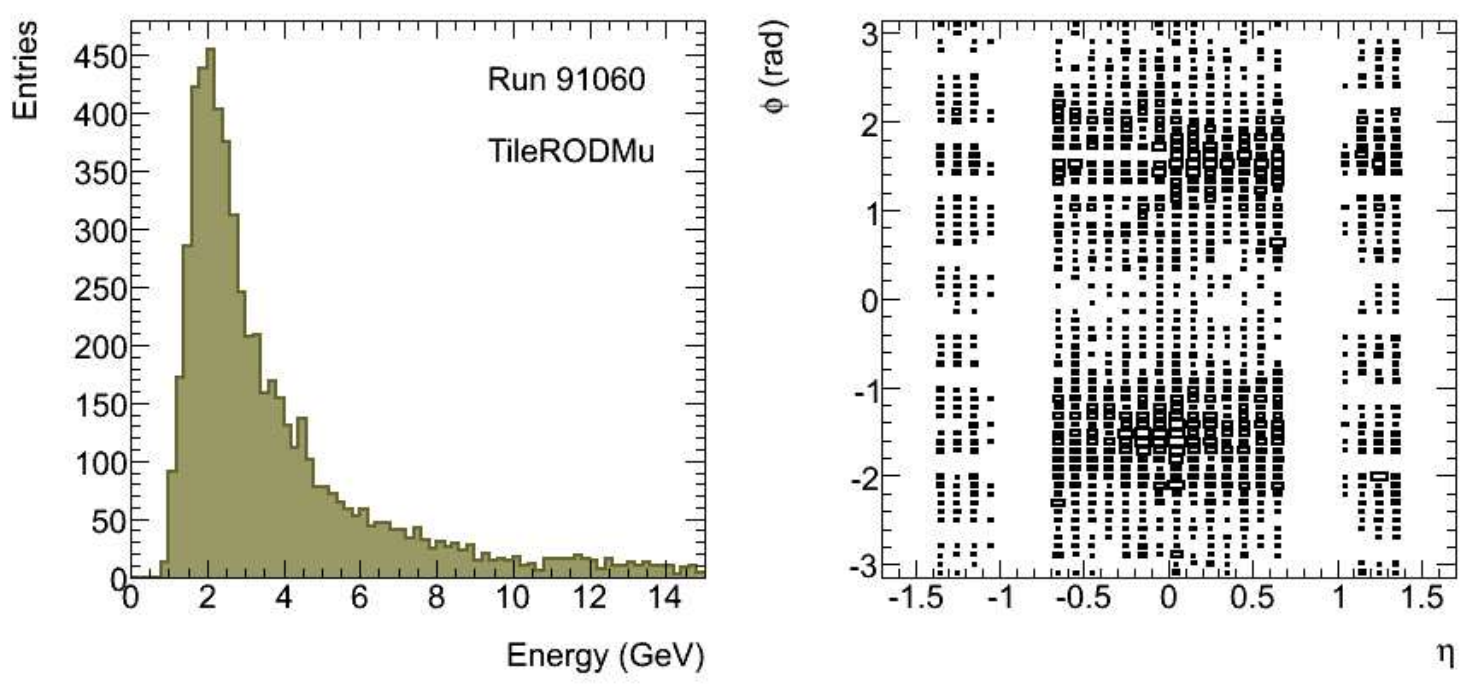

Figure 2: Reconstruction of cosmic events with muTile.

To refine the muFast $\mathrm{p}_{T}$, the algorithm muComb combines information from Inner Detector (ID) data, allowing to sharpen the threshold at low $\mathrm{p}_{T}$. Efficiencies for LVL2, with respect to LVL1, in the barrel is above $80 \%$ for muons with $\mathrm{p}_{T}$ at the selection edge and well above $90 \%$ for muons with higher $\mathrm{p}_{T}$.

Using information from calorimeter, muTile looks for an energy deposit compatible with the energy loss from muons. In the figure 2 shows the reconstructed energy and the $\eta$ vs $\phi$ position of cosmic events.

\section{Event Filter}

The Muon Event Filter consists of four algorithms: SegmentFinder, TrackBuilder, Extrapolator and Combiner. They are wrappers for the offline reconstruction tools. EF processing normally starts from the muFast result but, for debug purpose, can use LVL1 RoI directly. Segments are 
made first, using MDT precision hits. Tracks are made from segments, adding information from other muon detectors.

The extrapolation to the interaction point uses a parametrization of the energy loss in the calorimeter, for faster computation. Information from the inner detector EF algorithms is then added to make a combined track. The parameters of the tracks are finally obtained after refitting the hits actually used.

\subsection{Efficiency from $Z \rightarrow \mu \mu$ sample}

This method can be applied to extract MS trigger efficiency directly from data. One $\mu$ with $\mathrm{p}_{T}>20 \mathrm{GeV}$ and $|\eta|<2.4$ reconstructed in both ID and MS is used as a tag. The other $\mu$ requiring only the ID, is used as a probe. The separation between the two muons must be $\Delta \phi>0.3$ and the invariant mass must be close to the $Z$ mass $\left(\left|M_{\mu \mu}-M_{Z}\right|<10 \mathrm{GeV}\right)$. The threshold trigger efficiency is measured with good precision when compared to $\mathrm{MC}$ generator.

\subsection{Trigger Menus}

Different kinds of physics events need to share available bandwidth, that is limited. Flexible trigger menus allow to avoid saturation from few processes, and guarantee the possibility to organize the analysis depending from luminosity conditions. When the rates are too high, prescale factors (PS) can be applied to signatures, i.e. a PS of 100 on a certain signature means that only one event out of every 100 events triggered by this signature will be actually available at the next step.

To ensure a certain amount of events, is defined also the pass through (PT) mode. A PT of 100 on a certain signature means that out of every 100 events there must be at least one event passed to the next step

The events are flagged without rejection for HLT studies. Express streams, not prescaled, at fixed thresholds, are used for calibration and monitoring purposes. A possible Trigger Menu for LHC startup is shown in table 1 for different muon $\mathrm{p}_{T}$ thresholds.

\subsection{A trigger chain example}

Trigger software works with objects called Trigger Elements (TE). Feature Extraction Algorithms (FEX) are activated by input TE produced by previous trigger levels. FEX access the detector data and compute physical quantities, Features, that are then associated to the output TE. Selection is done in Hypothesis Algorithms, that validate or reject TE according to trigger menu requirements.

In-flight decays of pions and kaons are the main source of LVL1 trigger rate at low $\mathrm{p}_{T}$. One goal of the muon HLT is to reject such secondary muons while having high selection efficiency on prompt muons up to $\mathrm{p}_{T}$ of $6 \mathrm{GeV} / \mathrm{c}$.

A track from such decays appears with a kink, and the $\chi^{2}$ of the fit is worse than prompt muon tracks. All possible kinematic parameters and statistical techniques must be used in order to reject such tracks. 


\begin{tabular}{lccccc} 
& Level 1 & \multicolumn{2}{c}{ Level 2 } & \multicolumn{2}{c}{ Event Filter } \\
Signatures & & PS & PT & PS & PT \\
\hline mu4 & 1 & 5 & 400 & 300 & 0 \\
mu6 & 1 & 1 & 200 & 30 & 0 \\
mu10 & 1 & 1 & 100 & 1 & 0 \\
mu15 & 1 & 1 & 20 & 1 & 0 \\
mu20 & 1 & 1 & 0 & 1 & 0 \\
mu40 & 1 & 1 & 1 & 1 & 0 \\
mu20_passHLT & 1 & 1000 & 20 & 1 & 0 \\
2mu4 & 1 & 1 & 5 & 1 & 0 \\
2mu6 & 1 & 1 & 1 & 1 & 0 \\
mu4_mu6 & 1 & 1 & 1 & 1 & 0 \\
2mu10 & 1 & 1 & 0 & 1 & 0 \\
2mu20 & 1 & 1 & 1 & 1 & 0 \\
\hline
\end{tabular}

Table 1: A possible Muon Trigger startup Menu for $10^{31}$ luminosity.

\section{Conclusions}

ATLAS had a successful startup in 2008. The LHC is expected to restart in summer 2009 and ATLAS is looking forward to first p-p collisions soon afterwards. A big effort will be required to the trigger, to reach the needed performances of background suppression and high efficiency for events signal of new physics. The ATLAS Muon Trigger should be ready for ATLAS startup in 2009 .

\section{References}

[1] The ATLAS Collaboration, G. Aad et al., The ATLAS Experiment at the CERN Large Hadron Collider, JINST 3 (2008) S08003.

[2] ATLAS Collaboration, ATLAS High-Level Trigger Data Acquisition and Controls CERN/LHCC/2003-022, 2003

[3] ATLAS Muon HLT Review, [Online] https://twiki.cern.ch/twiki/bin/view/ Atlas/MuonHLTReview and references therein 